\title{
Characterization of Spacecraft and Debris Trajectory Stability within Binary Asteroid Systems
}

\author{
Eugene G. Fahnestock* \\ University of Michigan, Ann Arbor, Michigan, USA \\ Daniel J. Scheeres ${ }^{\dagger}$ \\ University of Colorado, Boulder, Colorado, USA
}

\begin{abstract}
Using $1999 \mathrm{KW} 4$ as a representative binary asteroid system, we simulate particles in the restricted full three body problem, subject to the complex time-varying (in any frame) gravity field of the binary components, evolving under the full two body problem. Trajectories are propagated for prograde and retrograde orbit initial conditions about each component and the system barycenter, varying initial orbit radius and inclination. For all particles we track impact and escape outcomes and compute finite time Lyapunov characteristic exponents as a metric of trajectory instability in this highly perturbed environment. For select orbit conditions we use an iterative scheme to refine the initial velocity in an attempt to improve trajectory duration and this metric. We synthesize results to globally map the degree of instability throughout the binary, relevant to execution of scientific missions to such a pair.
\end{abstract}

\section{Introduction}

$\mathrm{T}$ HE investigation of the stability and navigability of spacecraft trajectories about and within a binary asteroid system is becoming more relevant because the possibility of visiting such a pair in the near future, whether intentionally or accidentally, is growing. If any mission were mounted to a small (roughly kilometer sized) near-Earth asteroid (NEA) target with no prior indication of whether or not the target is a multiple system, there would be approximately a 1 in 7 chance that the target would be a binary based on current estimates for the binary fraction of the NEA population as $15 \pm 5 \% .{ }^{1-4}$ Thus the mission design and plan of operations must be prepared to adapt to that scenario.

Intentionally choosing to visit a binary NEA rather than accidentally doing so would be preferred, and is favorable relative to visiting a solitary NEA because of the increased information available. This includes remote determination of mass fraction, bulk density, and first moments of inertia with approximate body mass distribution (via recently published methods ${ }^{5}$ ) while still at a large distance on approach to the system. Once flying within the system, much more detailed internal mass distribution can be estimated, and extensive optical and spectrographic surface observations made, for two bodies rather than one with nearly the same mission cost. The fact that the pair will have different size and spin and likely structure and surface geology as well, controlling for the same heliocentric orbit evolution, will also yield many insights. In addition, the nature of binary systems may allow for very interesting dynamical experiments to be planned into the mission, such as creation of debris structures with small explosives and monitoring their evolution, occurring over faster time-scales than natural debris structures evolve.

In any case, navigating and controlling flight for a spacecraft within a typical binary NEA is challenging because the two rotating and co-orbiting, and possibly highly aspherical, bodies may exhibit exotic motions consistent with excited coupled dynamical configurations, like those explored in detail for the observed 1999 KW4 binary system. ${ }^{5-7}$ The gravity field is significantly time-varying in any chosen system frame, as is the

*Ph.D. Candidate, Department of Aerospace Engineering, University of Michigan, 2008 FXB Building, 1320 Beal Avenue, Ann Arbor, MI 48109, AIAA Student Member.

${ }^{\dagger}$ A. Richard Seebass Professor, Department of Aerospace Engineering Sciences, Colorado Center for Astrodynamics Research and University of Colorado Boulder, AIAA Associate Fellow. 
Jacobi function value, and the force environment felt by the spacecraft is characterized by large perturbations. However, these perturbations are also the prime source of structural information about the system itself.

The question of how to maximize the science return for a mission flying within a binary system entails a trade-off between that which helps get this information about the density distribution of the components, and helps increase the angular coverage of the components' surfaces by cameras and other instruments, vs. what helps keep the spacecraft safe and functional for a longer total flight time (i.e. longer observation time). For the former, it is preferable to allow the spacecraft to propagate with no trajectory actuation (yet have it go through many positions within the binary system, e.g. reach higher latitudes by a path with greater inclination and execute very low altitude passes). Whereas to increase spacecraft safety and mission lifetime it is preferable to do as many thruster firings as needed to ensure the spacecraft doesn't impact anywhere, while keeping the number of such firings as small as possible to minimize cumulative maneuver $\Delta V$. For this, it may instead be desirable to keep the spacecraft to low inclinations with respect to the binary mutual orbit (neglecting solar radiation pressure considerations). Several other engineering constraints must also be satisfied: maintaining line of sight to Earth, remaining in sunlight for the correct fraction of the time and with correct attitude, etc. The optimal design in the trade-offs involved depends critically on how easily and over what duration a spacecraft initially on an orbit of a certain type within a comparatively safe region in the binary will be removed from that region.

In this paper, we characterize the stability of orbits about and within a binary asteroid system that are relevant to the execution of scientific missions like that described above. Our principle tool is numerical propagation of individual test particles in the restricted yet "full" three body problem. That is, each massless particle has no influence on the motion of the binary itself but is subject to the full-detail gravity field of the primary (Alpha) and secondary (Beta) as those evolve according to the nontrivial full two body problem.

Subsequent sections of this paper are organized as follows. Our definition of the example binary system, our selection of its "substrate" simulated motion, our setup of the parameter space to be explored within the system, our propagation of the test particles, and our method for processing the trajectory results is all outlined in section II. Then we present the results of that study in section III. A simple iterative scheme aimed at improving the quality of the trajectories thus obtained through adjustment of the initial orbit velocity condition is described in section IV, with results shown in section V. We offer some conclusions about the design of trajectories in a binary within section VI.

\section{Methodology for Propagation and Stability Characterization of Trajectories}

The underlying full two body problem evolution of the binary itself is driven by the instantaneous mutual gravitational potential of the two (assumed rigid) celestial bodies involved. This mutual gravitational potential has been expressed using spherical harmonics. ${ }^{8,9}$ But, the harmonic expansion is not guaranteed to converge, much as the spherical harmonic expansion for the gravitational potential of a single body is in fact known to diverge at locations interior to the sphere of maximum extent surrounding that body. Due to other numerical issues, we also do not use a hull-filling grid of mass concentrations or similar methods of modeling the binary mutual potential. Instead we use a mutual potential formulation based on polyhedral body models ${ }^{10}$ as previously implemented for binary system propagation. ${ }^{11}$

We use the system model for the earlier mentioned KW4 system in particular, because it is still one of the best characterized binary NEAs (and further, PHAs) to date. It is also a representative example of the largest class of binary NEAs, characterized by a larger, roughly axisymmetric primary spinning significantly faster than the synchronous rate, and a smaller roughly triaxial or asymmetric secondary locked into onaverage synchronous rotation. The full two body problem motion results set used is one with the highest plausible excitation level identified for KW4, being the "most interesting" and most severely perturbing to the test particle motion. In this configuration the initial spin-orbit pole offset angle for the system's Cassini state is $10^{\circ}$ and the initial eccentricity and mean anomaly are 0.01 and $180^{\circ} .5$ The state of the binary system itself is interpolated from this most-excited case's binary system propagation output files, whenever required for computation of the net force acting on each test particle. That net force is determined as the sum of the forces due to the gravity of each polyhedral model, computed by an established algorithm. ${ }^{12}$

We examine nominal trajectories in six regions of the position and velocity phase space for this system: both prograde and retrograde close orbits about Alpha, prograde and retrograde close orbits about Beta, and prograde and retrograde far-field orbits about the system barycenter. Within each such combination 
of orbit region and type, nominal trajectory initial states are chosen to sample both radius and inclination, the latter with values of $\left\{0^{\circ}, 10^{\circ}, 20^{\circ}, 30^{\circ}, 40^{\circ}\right\}$. In all cases the initial position is placed along the extended line of syzygy, measuring the radius value from the base point (centroid or barycenter, as appropriate) out along the direction of a vector pointing from Alpha toward Beta. For the radius value we use lower bounds of $110 \%$ of the maximum radius of any vertex point on the mesh of the body and upper bounds of $80 \%$ of the distance from the body centroid to the usual restricted three body problem L1 point along the line of syzygy (the line between the two body centroids). Alternatively, for the far-field orbits we use bounds of 2.0 and 3.0 times the initial centroids separation distance. Therefore the normalized radius values sampled for each of the region and type combinations are as indicated in Table 1. The initial velocity magnitude is

Table 1. Normalized $(1=2540.5 \mathrm{~m})$ initial orbital radius values sampled, for each region and type

\begin{tabular}{c|c|c}
\hline \hline $\begin{array}{c}\text { Alpha-centric, } \\
\text { prograde and retrograde }\end{array}$ & $\begin{array}{c}\text { Beta-centric, } \\
\text { prograde and retrograde }\end{array}$ & $\begin{array}{c}\text { Barycentric, } \\
\text { prograde and retrograde }\end{array}$ \\
\hline 0.3397 & 0.1415 & 2.02 \\
0.39443 & 0.12821 & 2.2222 \\
0.44916 & 0.15479 & 2.424 \\
0.50389 & 0.16808 & 2.626 \\
0.55862 & 0.18136 & 2.828 \\
0.61335 & 0.19465 & 3.03 \\
\hline \hline
\end{tabular}

chosen as the circular speed using the mass of the body being orbited about or mass of the whole system, as appropriate. The velocity vector with this magnitude is perpendicular to the line of syzygy and simply inclined w.r.t. the plane of the initial binary mutual orbit by the inclination value.

A nominal trajectory is found by propagating a single test particle from the initial condition of each regiontype-radius-inclination case (with 180 cases in all). The states are propagated in an inertial barycentric frame, using a Runge-Kutta Fehlberg 7(8) integrator, with the forces on the particle due to each binary component's mass distribution being computed in the respective body-fixed frame and then transformed to the integration frame. About each such nominal trajectory a small $(N \approx 20)$ Monte-Carlo batch of particles with initial conditions deviated in Gaussian random fashion from the initial condition of the nominal trajectory is also propagated. The perturbations are scaled to approximately the same size as rule-of-thumb spacecraft state uncertainty in an operational scenario $(1 \sigma=10 \mathrm{~m}$ in position, $1 \mathrm{~cm} / \mathrm{s}$ in velocity).

The final disposition (in terms of impact onto either body or escape from the system or remaining within the starting region even after a full two weeks of simulation duration) is tracked for the nominal trajectory and for all deviated initial condition particles in all batches of such particles. These outcomes are sorted by whether they are expected or not, according to comparison between the initial value of the (time-varying) Jacobi function for the trajectory and the L1 or L1-analogue Jacobi function values matching some idealized geometry models of the system. In particular, if the binary system were to be modeled as a spherical primary and a synchronously locked ellipsoidal secondary, then the Jacobi function would be constant over time, the Jacobi integral, allowing definition of zero velocity curves and definition of the point analogous to the L1 point in the usual restricted three body problem. The Jacobi value at this point can be used a a threshold value, such that all particles with actual system model initial time-varying Jacobi function value less than this threshold value are expected to have a final disposition of impact onto the binary component being orbited. Likewise, a Jacobi integral value of zero can be used as an upper threshold. Meaning escape becomes possible, though still not expected, for particles with an initial value for the actual system model time-varying Jacobi function that is greater than zero. The breakdown of actual particle outcomes vs. the expected particle outcomes from comparison of the initial Jacobi function value with the threshold values helps characterize the degree to which the time-varying nature of the dynamics is important for predicting the eventual outcome of a particle's motion.

Our main result for stability and lifetime in orbit, however, is computation of the finite-time Lyapunov Characteristic Exponent (LCE) for every particle. Along with each nominal or perturbed trajectory $\mathbf{x}$, we propagate an extremely close shadow trajectory $\mathbf{x}_{s}$, initially offset from $\mathbf{x}$ by a deviation $\delta \mathbf{x}$ of one millimeter 
in initial position and nothing in velocity. These are used in the following to get the LCE at a finite time $t$ :

$$
\xi_{t}=\frac{1}{t} \ln \left(\frac{\left\|\mathbf{x}_{s}\left(t ; \mathbf{x}_{o}+\delta \mathbf{x}, t_{o}\right)-\mathbf{x}\left(t ; \mathbf{x}_{o}, t_{o}\right)\right\|}{\|\delta \mathbf{x}\|}\right)
$$

Larger positive values indicate greater instability. The LCE vs. time curves (or the value of the peak, time-integral average, or final value, by order of increasing importance, for these curves) can be compared within a batch of perturbed particles. The nominal trajectory's LCE vs. time curve, or the combined such curve for all nominal and perturbed particles found by averaging at every plotted time, or just the average of the LCE values for all nominal and perturbed particles at one given cutoff time, can all be compared between batches. This yields comparison across initial radius and inclination conditions and regions and types of orbits, to globally map the degree of instability throughout the system. This fulfills the primary objective of this paper.

In theory, we could also map the representative final LCE value for each perturbed particle onto the planes in phase space in which the initial state perturbations from the nominal trajectory were taken. This would allow us to ascertain gradients in stability characteristics along each dimension of phase space, in the extreme locality of each case in our parameter space. However, the number of perturbed particles needs to be higher than the $\approx 20$ used herein, in order for this gradient information to be sufficiently revealed.

\section{Global Results}

As an example of the unprocessed output of the particle propagations, the nominal and perturbed particle trajectories are plotted in the barycentric inertial frame for one case - the Alpha-centric prograde orbit at 0.50389 normalized radius and $0^{\circ}$ inclination - within Figure 1. These trajectories have their corresponding

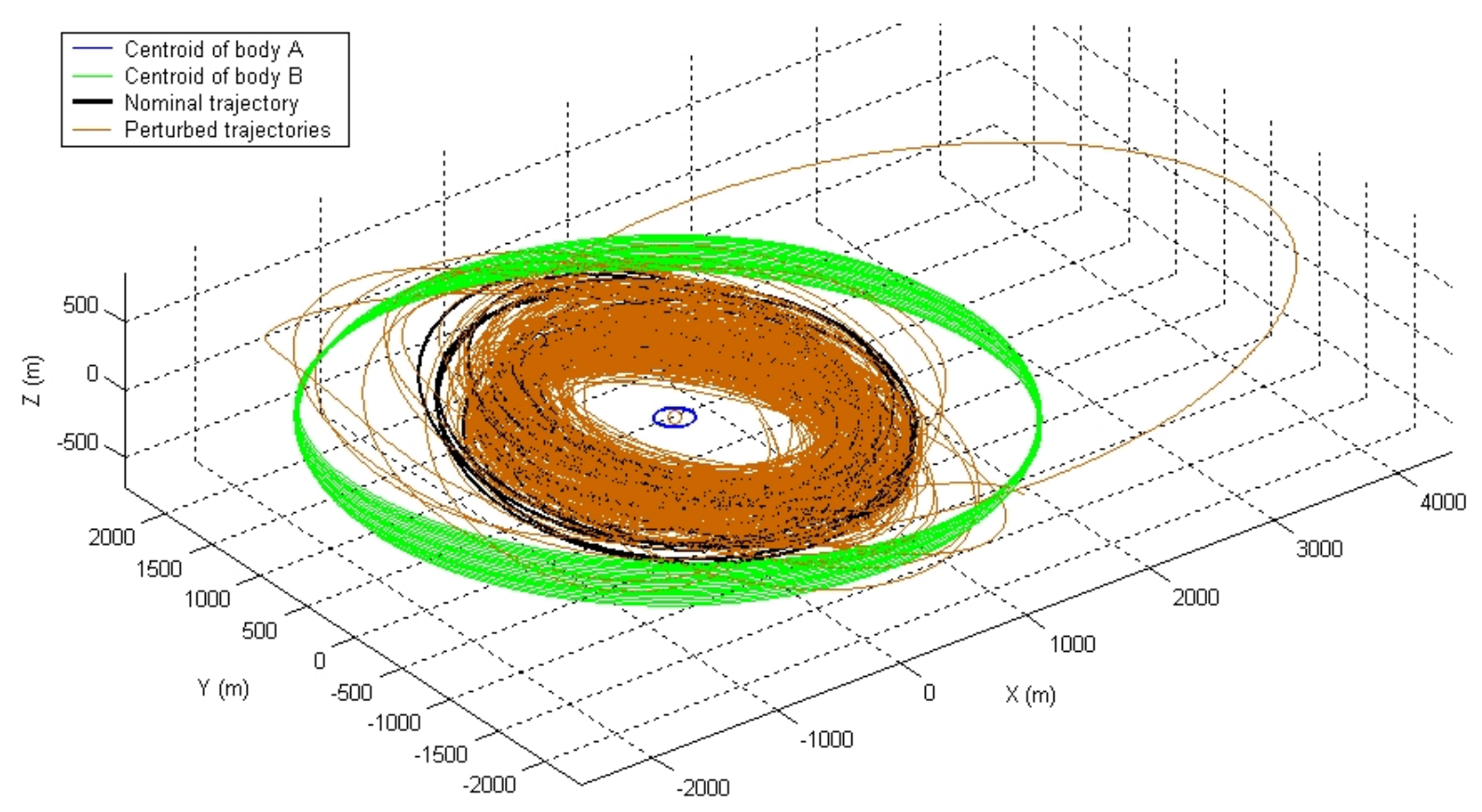

Figure 1. Nominal and perturbed trajectories plotted in barycentric inertial space for Alpha-centric, prograde, $R_{o}=0.50389,0^{\circ}$ inclination case.

LCE vs. time curves shown in the fourth (center right) panel of Figure 2. What this Figure 2 reveals is the difference in spacecraft or particle lifetime and behavior across cases in the same region (about Alpha) and with the same orbit type (prograde) and same inclination $\left(0^{\circ}\right)$ but varying the initial radius parameter from small to large. In addition to the nominal trajectory being plotted with a thick black solid line, the thin black dashed lines give the extent of the previous one-step smaller in radius LCE vs. time curves. 


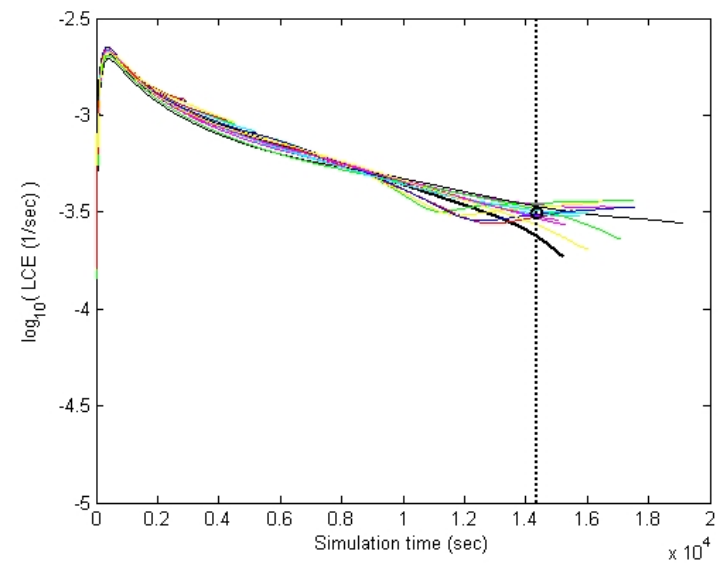

(a) $R_{o}=0.3397$

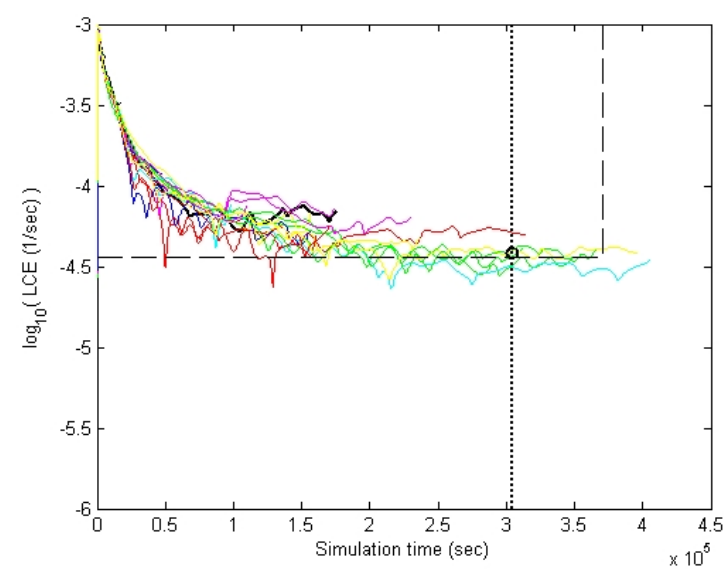

(c) $R_{o}=0.44916$

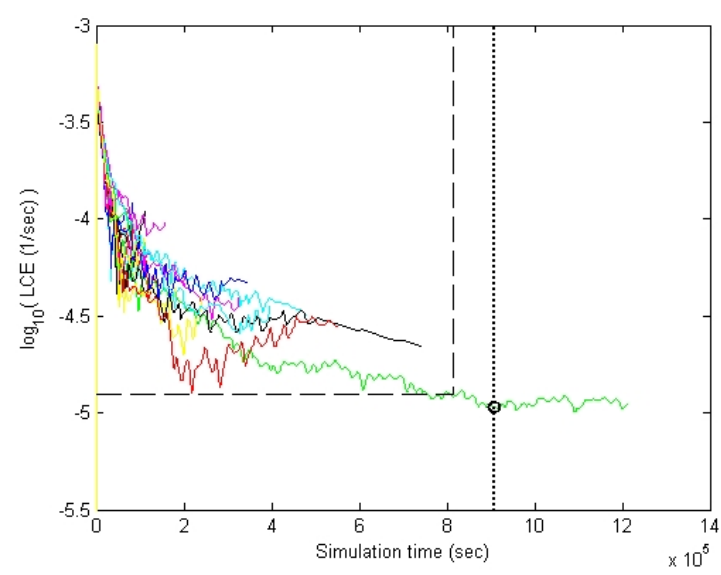

(e) $R_{o}=0.55862$

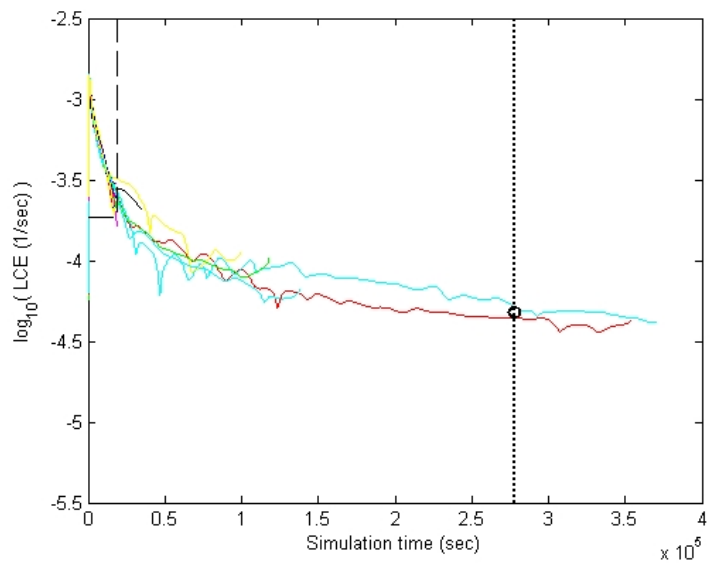

(b) $R_{o}=0.39443$

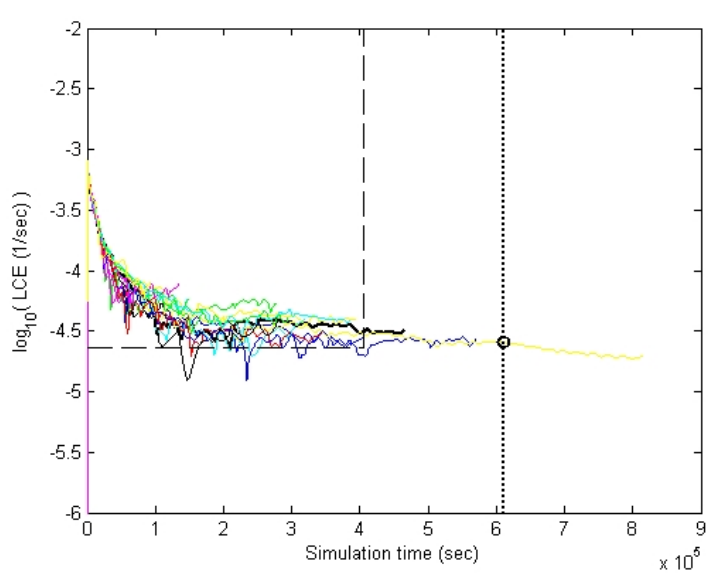

(d) $R_{o}=0.50389$

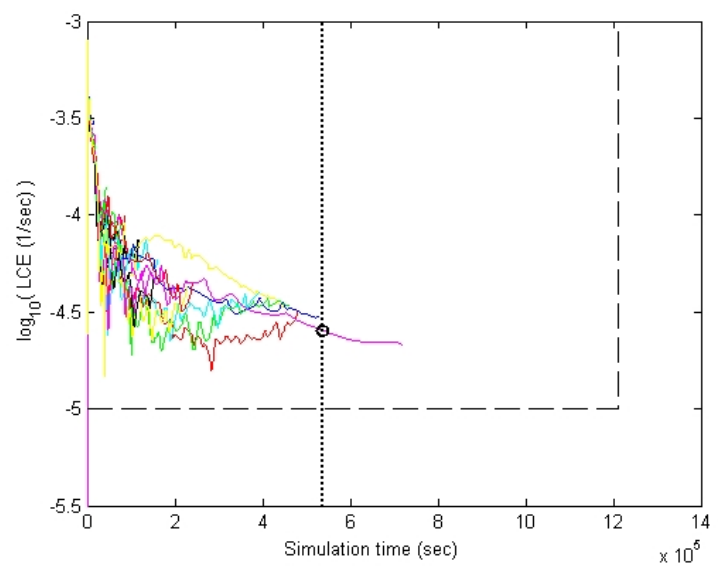

(f) $R_{o}=0.61335$

Figure 2. Values of the LCE plotted against simulation time, for all Alpha-centric, prograde, $0^{\circ}$ inclination cases, with increasing $R_{o}$ from top left panel. In each case, the nominal trajectory is shown with a thick black solid line, perturbed trajectories are shown with the thin colored solid lines, the bounds on the previous panel's curves (if applicable) are shown with the thin black dashed lines, and the cutoff time for evaluating the average LCE value (marked) is shown with the thick black dotted line. 
By extent, we mean the region bounded at right by the longest particle flight duration and bounded from below by the minimum particle LCE value (obviously excepting the very initial period). For each case, we arbitrarily select $25 \%$ less than the longest particle flight duration as a cutoff time at which to evaluate the dispositions of the particles, and at which to interpolate and average the LCE values for those particles still having the "lingering in orbit" disposition at that cutoff time. The cutoff time is indicated in Figure 2 by the thick black dotted line in each panel. The average of the LCE values for the still in orbit particles at the cutoff time is shown on the thick black dotted line by a circle marker. This average LCE value information is accumulated across many cases in Table 2 and in Figure 3, in the $\log _{10}$ (avg. LCE) column and in the height of the bars/pillars, respectively. The cutoff times are also listed in the appropriate column and used for the color coding of the bar/pillars in the plot.

Table 2. Simulated particle dispositions and LCE values for Alpha-centric, prograde cases:

\begin{tabular}{|c|c|c|c|c|c|c|c|}
\hline$R_{o}(\mathrm{DU})$ & $i_{o}\left(^{\circ}\right)$ & $\begin{array}{c}\text { cutoff time } \\
\text { (d:hh:mm:ss) }\end{array}$ & $\alpha^{*}(\%)$ & $\beta^{*}(\%)$ & Esc.* $(\%)$ & Flight* (\%) & $\log _{10}$ (avg. LCE) \\
\hline 0.3397 & 0 & $0: 03: 59: 31$ & $7(35)$ & $0(0)$ & $0(0)$ & $13(65)$ & -3.5075 \\
\hline 0.3397 & 10 & $0: 05: 21: 25$ & $17(85)$ & $0(0)$ & $0(0)$ & $3(15)$ & -3.5927 \\
\hline 0.3397 & 20 & 0:07:11:07 & $18(90)$ & $0(0)$ & $0(0)$ & $2(10)$ & -3.7151 \\
\hline 0.3397 & 30 & 1:09:45:56 & $18(90)$ & $0(0)$ & $0(0)$ & $2(10)$ & -4.1239 \\
\hline 0.3397 & 40 & $1: 07: 37: 21$ & $19(95)$ & $0(0)$ & $0(0)$ & $1(5)$ & -4.0465 \\
\hline 0.39443 & 0 & $3: 05: 14: 49$ & $18(90)$ & $0(0)$ & $0(0)$ & $2(10)$ & -4.3169 \\
\hline 0.39443 & 10 & $2: 02: 7: 55$ & $15(75)$ & $2(10)$ & $0(0)$ & $3(15)$ & -4.115 \\
\hline 0.39443 & 20 & $5: 20: 43: 55$ & $16(80)$ & $2(10)$ & $0(0)$ & $2(10)$ & -4.6408 \\
\hline 0.39443 & 30 & $4: 17: 40: 39$ & $18(90)$ & $0(0)$ & $0(0)$ & $2(10)$ & -4.4241 \\
\hline 0.39443 & 40 & $10: 12: 25: 25$ & $16(80)$ & $3(15)$ & $0(0)$ & $1(5)$ & -5.0003 \\
\hline 0.44916 & 0 & $3: 12: 27: 26$ & $12(60)$ & $2(10)$ & $0(0)$ & $6(30)$ & -4.4166 \\
\hline 0.44916 & 10 & $5: 05: 41: 06$ & $14(70)$ & $4(20)$ & $0(0)$ & $2(10)$ & -4.486 \\
\hline 0.44916 & 20 & $6: 20: 22: 58$ & $13(65)$ & $5(25)$ & $0(0)$ & $2(10)$ & -4.6629 \\
\hline 0.44916 & 30 & $6: 11: 52: 53$ & $12(60)$ & $3(15)$ & $0(0)$ & $5(25)$ & -4.6318 \\
\hline 0.44916 & 40 & 10:12:25:25 & $18(90)$ & $1(5)$ & $0(0)$ & $1(5)$ & -4.7834 \\
\hline 0.50389 & 0 & $7: 01: 51: 41$ & $15(75)$ & $4(20)$ & $0(0)$ & $1(5)$ & -4.5954 \\
\hline 0.50389 & 10 & $6: 20: 27: 07$ & $12(60)$ & $6(30)$ & $0(0)$ & $2(10)$ & -4.5795 \\
\hline 0.50389 & 20 & $10: 12: 25: 25$ & $10(50)$ & $7(35)$ & $2(10)$ & $1(5)$ & -4.806 \\
\hline 0.50389 & 30 & $10: 12: 25: 25$ & $13(65)$ & $5(25)$ & $0(0)$ & $2(10)$ & -4.7888 \\
\hline 0.50389 & 40 & $10: 12: 25: 25$ & $8(40)$ & $0(0)$ & $0(0)$ & $12(60)$ & -4.9291 \\
\hline 0.55862 & 0 & $10: 12: 25: 25$ & $13(65)$ & $6(30)$ & $0(0)$ & $1(5)$ & -4.9692 \\
\hline 0.55862 & 10 & 9:23:32:09 & $14(70)$ & $5(25)$ & $0(0)$ & $1(5)$ & -4.848 \\
\hline 0.55862 & 20 & $10: 12: 25: 25$ & $14(70)$ & $5(25)$ & $1(5)$ & $0(0)$ & -4.7876 \\
\hline 0.55862 & 30 & $10: 12: 25: 25$ & $12(60)$ & $3(15)$ & $0(0)$ & $5(25)$ & -4.7731 \\
\hline 0.55862 & 40 & 10:12:25:25 & $9(45)$ & $1(5)$ & $0(0)$ & $10(50)$ & -4.8722 \\
\hline 0.61335 & 0 & $6: 05: 37: 24$ & $12(60)$ & $7(35)$ & $0(0)$ & $1(5)$ & -4.5952 \\
\hline 0.61335 & 10 & $10: 12: 25: 25$ & $9(45)$ & $9(45)$ & $1(5)$ & $1(5)$ & -4.8668 \\
\hline 0.61335 & 20 & $10: 12: 25: 25$ & $14(70)$ & $4(20)$ & $2(10)$ & $0(0)$ & -4.8524 \\
\hline 0.61335 & 30 & $10: 12: 25: 25$ & $14(70)$ & $4(20)$ & $0(0)$ & $2(10)$ & -4.7727 \\
\hline 0.61335 & 40 & $10: 12: 25: 25$ & $11(55)$ & $4(20)$ & $0(0)$ & $5(25)$ & -4.7696 \\
\hline
\end{tabular}

* Refers to the number (and percentage) of particles reaching this outcome before the duration indicated by the "cutoff time" column has passed. The outcomes are impact onto Alpha, impact onto Beta, escape from the system as a whole, and remaining in flight, from left to right and considered as of the cutoff time. 


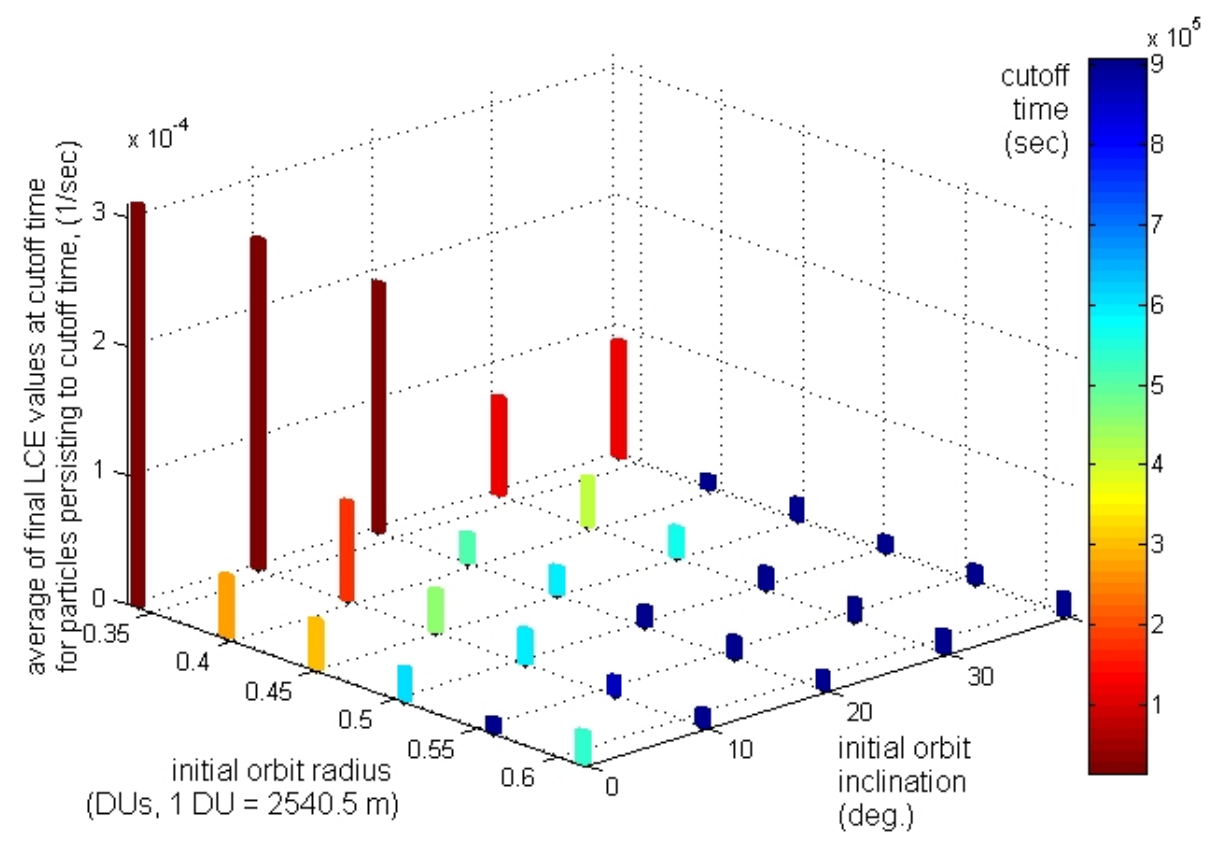

Figure 3. Graphical illustration of cutoff duration and average LCE value at that duration for Alpha-centric, prograde cases.

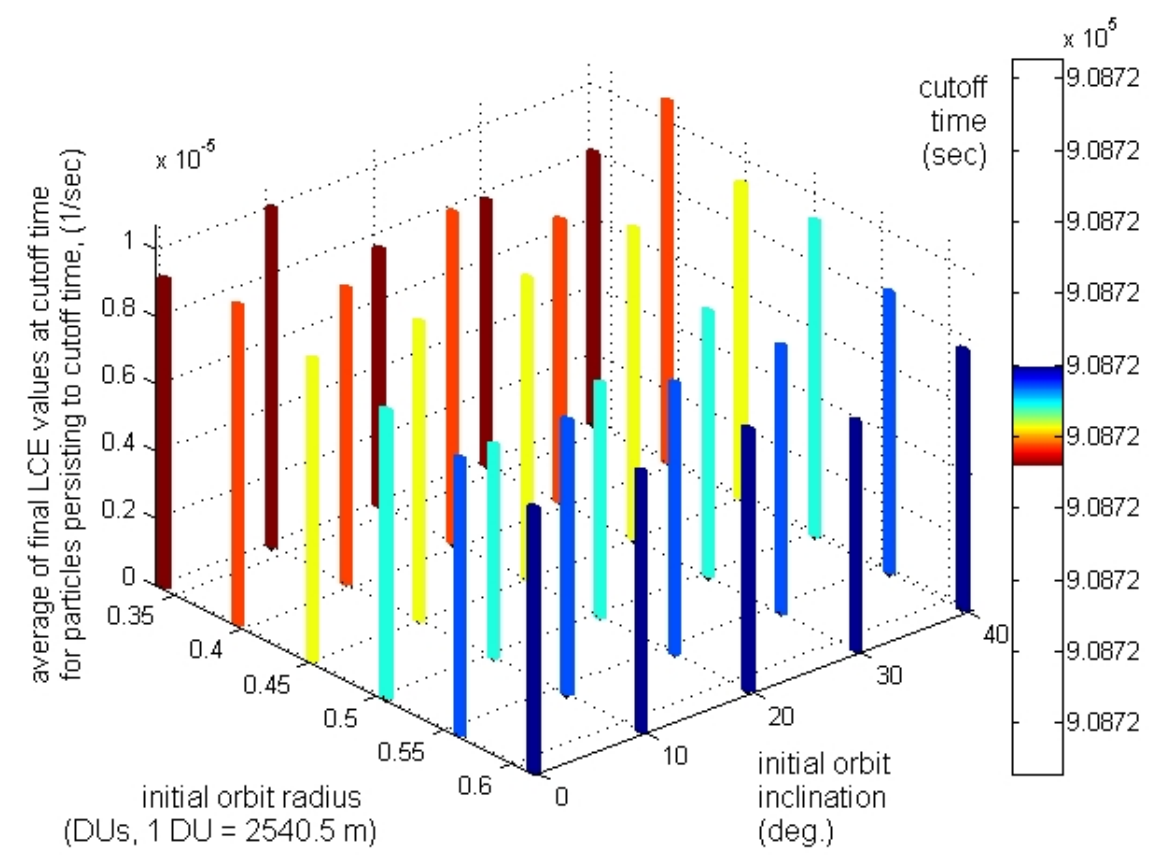

Figure 4. Graphical illustration of cutoff duration and average LCE value at that duration for Alpha-centric, retrograde cases.

Similar information is captured for the retrograde orbits about Alpha within Table 3 and in Figure 4. Note the color scale in Figure 4 really indicates that that all of these cases have the same value for cutoff duration, yet the colors are differentiated between the initial radii for clarity. The uniform cutoff time is consistent with having at least one particle reach the maximum possible duration, limited to the length of the underlying full two body problem propagation, for every retrograde initial orbital condition about Alpha. 
Table 3. Simulated particle dispositions and LCE values for Alpha-centric, retrograde cases:

\begin{tabular}{r|r|r|c|c|c|c|r}
\hline \hline & & cutoff time & & & & \\
$R_{o}(\mathrm{DU})$ & $i_{o}\left(^{\circ}\right)$ & $(\mathrm{d}: \mathrm{hh}: \mathrm{mm}: \mathrm{ss})$ & $\alpha^{*}(\%)$ & $\beta^{*}(\%)$ & Esc.* $(\%)^{*}$ & Flight* $(\%)$ & $\log _{10}$ (avg. LCE $)$ \\
\hline 0.3397 & 0 & $10: 12: 25: 25$ & $19(90)$ & $0(0)$ & $0(0)$ & $2(10)$ & -5.0379 \\
0.3397 & 10 & $10: 12: 25: 25$ & $20(95)$ & $0(0)$ & $0(0)$ & $1(5)$ & -4.9982 \\
0.3397 & 20 & $10: 12: 25: 25$ & $17(81)$ & $0(0)$ & $0(0)$ & $4(19)$ & -5.1163 \\
0.3397 & 30 & $10: 12: 25: 25$ & $12(57)$ & $0(0)$ & $0(0)$ & $9(43)$ & -5.1047 \\
0.3397 & 40 & $10: 12: 25: 25$ & $12(57)$ & $0(0)$ & $0(0)$ & $9(43)$ & -5.092 \\
\hline 0.39443 & 0 & $10: 12: 25: 25$ & $15(94)$ & $0(0)$ & $0(0)$ & $1(6)$ & -5.0237 \\
0.39443 & 10 & $10: 12: 25: 25$ & $13(93)$ & $0(0)$ & $0(0)$ & $1(7)$ & -5.0561 \\
0.39443 & 20 & $10: 12: 25: 25$ & $12(92)$ & $0(0)$ & $0(0)$ & $1(8)$ & -5.0079 \\
0.39443 & 30 & $10: 12: 25: 25$ & $11(92)$ & $0(0)$ & $0(0)$ & $1(8)$ & -5.0759 \\
0.39443 & 40 & $10: 12: 25: 25$ & $10(91)$ & $0(0)$ & $0(0)$ & $1(9)$ & -4.9704 \\
\hline 0.44916 & 0 & $10: 12: 25: 25$ & $8(73)$ & $2(18)$ & $0(0)$ & $1(9)$ & -5.0471 \\
0.44916 & 10 & $10: 12: 25: 25$ & $6(55)$ & $4(36)$ & $0(0)$ & $1(9)$ & -5.0521 \\
0.44916 & 20 & $10: 12: 25: 25$ & $9(82)$ & $1(9)$ & $0(0)$ & $1(9)$ & -5.0472 \\
0.44916 & 30 & $10: 12: 25: 25$ & $9(82)$ & $1(9)$ & $0(0)$ & $1(9)$ & -5.0343 \\
0.44916 & 40 & $10: 12: 25: 25$ & $11(92)$ & $0(0)$ & $0(0)$ & $1(8)$ & -5.0277 \\
\hline 0.50389 & 0 & $10: 12: 25: 25$ & $0(0)$ & $0(0)$ & $0(0)$ & $21(100)$ & -5.0673 \\
0.50389 & 10 & $10: 12: 25: 25$ & $6(55)$ & $4(36)$ & $0(0)$ & $1(9)$ & -5.1993 \\
0.50389 & 20 & $10: 12: 25: 25$ & $5(45)$ & $5(45)$ & $0(0)$ & $1(9)$ & -5.1582 \\
0.50389 & 30 & $10: 12: 25: 25$ & $7(64)$ & $3(27)$ & $0(0)$ & $1(9)$ & -5.1041 \\
0.50389 & 40 & $10: 12: 25: 25$ & $8(73)$ & $2(18)$ & $0(0)$ & $1(9)$ & -5.0292 \\
\hline 0.55862 & 0 & $10: 12: 25: 25$ & $0(0)$ & $0(0)$ & $0(0)$ & $21(100)$ & -5.0852 \\
0.55862 & 10 & $10: 12: 25: 25$ & $0(0)$ & $0(0)$ & $0(0)$ & $21(100)$ & -5.0882 \\
0.55862 & 20 & $10: 12: 25: 25$ & $0(0)$ & $0(0)$ & $0(0)$ & $21(100)$ & -5.0944 \\
0.55862 & 30 & $10: 12: 25: 25$ & $0(0)$ & $0(0)$ & $0(0)$ & $21(100)$ & -5.0996 \\
0.55862 & 40 & $10: 12: 25: 25$ & $1(5)$ & $0(0)$ & $0(0)$ & $20(95)$ & -5.0795 \\
\hline 0.61335 & 0 & $10: 12: 25: 25$ & $0(0)$ & $0(0)$ & $0(0)$ & $21(100)$ & -5.1038 \\
0.61335 & 10 & $10: 12: 25: 25$ & $0(0)$ & $0(0)$ & $0(0)$ & $21(100)$ & -5.1118 \\
0.61335 & 20 & $10: 12: 25: 25$ & $0(0)$ & $0(0)$ & $0(0)$ & $21(100)$ & -5.1671 \\
0.61335 & 30 & $10: 12: 25: 25$ & $0(0)$ & $0(0)$ & $0(0)$ & $21(100)$ & -5.1113 \\
0.61335 & 40 & $10: 12: 25: 25$ & $0(0)$ & $0(0)$ & $0(0)$ & $21(100)$ & \\
\hline \hline & & & & & & & \\
\hline
\end{tabular}

* See note on Table 2.

Note that the vertical scales are an order of magnitude different between Figures 3 and 4, which reveals how much more stable the retrograde Alpha-centric orbits are.

For the initial orbital conditions about Beta, both prograde and retrograde, similar information is shown in Table 4 and in Figure 5 for just the smallest radius trajectories (those passing closest to Beta's surface). At the opposite extreme in terms of separation from all binary component bodies, the far-field orbits about the binary barycenter at the maximum radius value sampled have cutoff times and average LCE values at the cutoff times as shown in Table 5 and represented in Figure 6.

From the results presented herein, we find that in general, among the Alpha-centric orbits, retrograde orbital paths are more robust to the gravity perturbations of the binary system and enjoy longer lifetimes and lower cutoff LCE values than prograde orbital paths. Indeed, all of the retrograde orbital paths saturate at the maximum possible duration given our methodology. This is sensible, as the more rapid relative rotation rate of the spacecraft with respect to Alpha's surface accomplishes a better averaging of the non- 
Table 4. Simulated particle dispositions and LCE values for Beta-centric, prograde and retrograde cases:

PROGRADE

\begin{tabular}{|c|c|c|c|c|c|c|c|}
\hline$R_{o}(\mathrm{DU})$ & $i_{o}\left(^{\circ}\right)$ & $\begin{array}{l}\text { cutoff time } \\
\text { (d:hh:mm:ss) }\end{array}$ & $\alpha^{*}(\%)$ & $\beta^{*}(\%)$ & Esc. ${ }^{*}(\%)$ & Flight* $(\%)$ & $\log _{10}$ (avg. LCE) \\
\hline 0.1415 & 0 & $0: 01: 30: 45$ & $0(0)$ & $20(95)$ & $0(0)$ & $1(5)$ & -3.1878 \\
\hline 0.1415 & 10 & 0:04:44:21 & $0(0)$ & $19(90)$ & $0(0)$ & $2(10)$ & -3.5681 \\
\hline 0.1415 & 20 & $10: 12: 25: 25$ & $0(0)$ & $20(95)$ & $0(0)$ & $1(5)$ & -4.8562 \\
\hline 0.1415 & 30 & 0:01:30:02 & $0(0)$ & $15(71)$ & $0(0)$ & $6(29)$ & -3.1124 \\
\hline 0.1415 & 40 & 0:09:02:14 & $0(0)$ & $20(95)$ & $0(0)$ & $1(5)$ & -3.8013 \\
\hline \multicolumn{8}{|c|}{ RETROGRADE } \\
\hline 0.1415 & 0 & 0:01:28:09 & $0(0)$ & $19(90)$ & $0(0)$ & $2(10)$ & -3.1201 \\
\hline 0.1415 & 10 & 10:06:53:10 & $0(0)$ & $20(95)$ & $0(0)$ & $1(5)$ & -4.8336 \\
\hline 0.1415 & 20 & $0: 10: 14: 17$ & $0(0)$ & $20(95)$ & $0(0)$ & $1(5)$ & -3.8927 \\
\hline 0.1415 & 30 & $5: 04: 38: 59$ & $0(0)$ & $20(95)$ & $0(0)$ & $1(5)$ & -4.6748 \\
\hline 0.1415 & 40 & 0:09:32:57 & $0(0)$ & $20(95)$ & $0(0)$ & $1(5)$ & -3.8062 \\
\hline
\end{tabular}

* See note on Table 2.

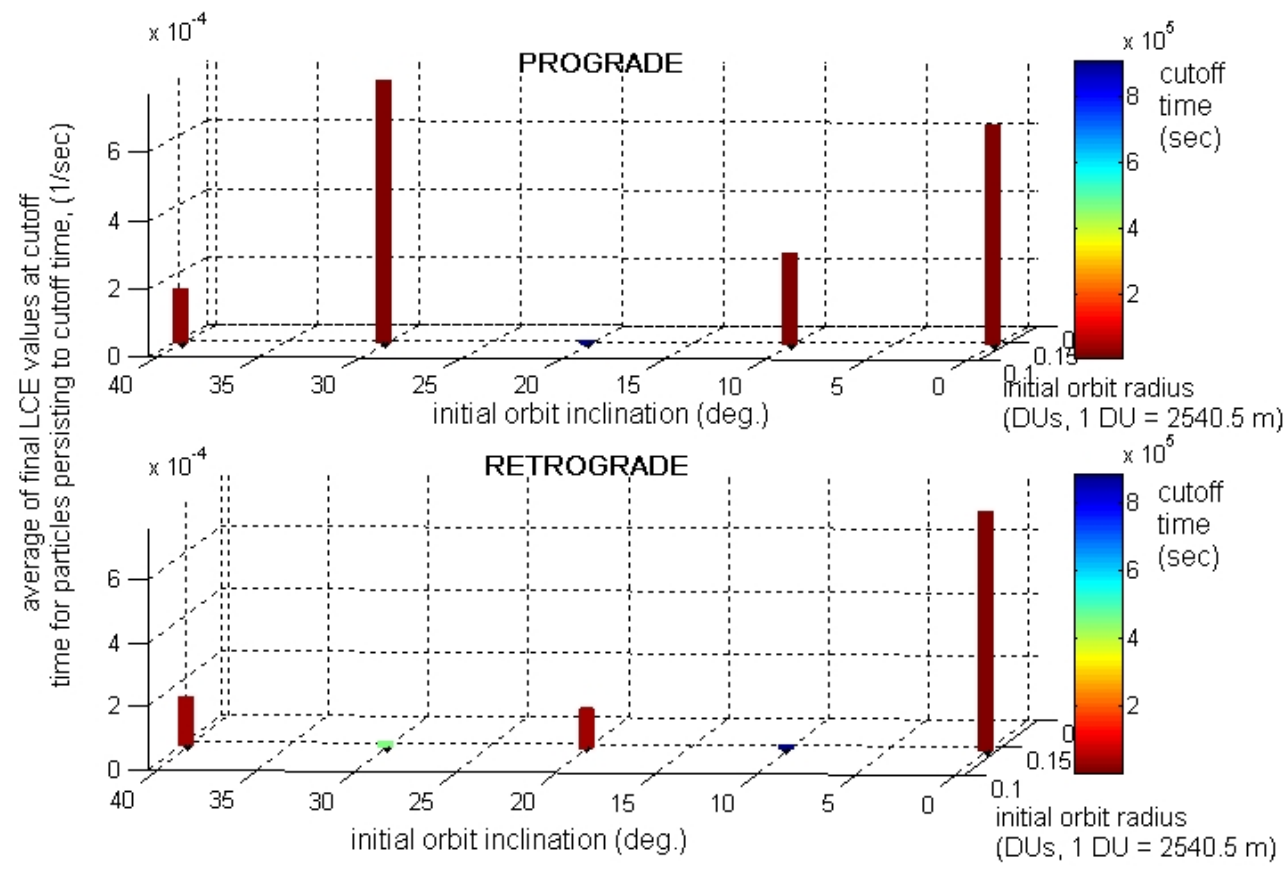

Figure 5. Graphical illustration of cutoff duration and average LCE value at that duration for Beta-centric orbits, both prograde and retrograde, passing closest to Beta's surface.

axisymmetric components of Alpha's gravity field. The same principle applies for effectively better averaging out of the gravitational perturbation from Beta. The retrograde paths also incidentally improve the rate of angular coverage for imaging or similar mapping observations of Alpha's surface.

We also find that higher inclination tends to promote longer lifetime in each case, especially for the prograde orbits. This is somewhat surprising, as we might expect that Alpha's oblateness and the gravitational interaction with Beta would more rapidly grow the eccentricity of more highly inclined orbits, producing impact with Alpha sooner rather than later. A richer set of analysis already exists for three body problem 
Table 5. Simulated particle dispositions and LCE values for barycentric, prograde and retrograde cases:

PROGRADE

\begin{tabular}{|c|c|c|c|c|c|c|c|}
\hline$R_{o}(\mathrm{DU})$ & $i_{o}\left({ }^{\circ}\right)$ & $\begin{array}{c}\text { cutoff time } \\
\text { (d:hh:mm:ss) }\end{array}$ & $\alpha^{*}(\%)$ & $\beta^{*}(\%)$ & Esc. ${ }^{*}(\%)$ & Flight* (\%) & $\log _{10}$ (avg. LCE) \\
\hline 3.03 & 0 & $10: 12: 25: 25$ & $0(0)$ & $0(0)$ & $0(0)$ & $21(100)$ & -5.3689 \\
\hline 3.03 & 10 & $10: 12: 25: 25$ & $0(0)$ & $0(0)$ & $0(0)$ & $21(100)$ & -5.3594 \\
\hline 3.03 & 20 & $10: 12: 25: 25$ & $0(0)$ & $0(0)$ & $0(0)$ & $21(100)$ & -5.3516 \\
\hline 3.03 & 30 & $10: 12: 25: 25$ & $0(0)$ & $0(0)$ & $0(0)$ & $21(100)$ & -5.3648 \\
\hline 3.03 & 40 & $10: 12: 25: 25$ & $0(0)$ & $0(0)$ & $0(0)$ & $21(100)$ & -5.3492 \\
\hline \multicolumn{8}{|c|}{ RETROGRADE } \\
\hline 3.03 & 0 & $10: 12: 25: 25$ & $0(0)$ & $0(0)$ & $0(0)$ & $21(100)$ & -5.3604 \\
\hline 3.03 & 10 & $10: 12: 25: 25$ & $0(0)$ & $0(0)$ & $0(0)$ & $21(100)$ & -5.3546 \\
\hline 3.03 & 20 & $10: 12: 25: 25$ & $0(0)$ & $0(0)$ & $0(0)$ & $21(100)$ & -5.3641 \\
\hline 3.03 & 30 & $10: 12: 25: 25$ & $0(0)$ & $0(0)$ & $0(0)$ & $21(100)$ & -5.3562 \\
\hline 3.03 & 40 & $10: 12: 25: 25$ & $0(0)$ & $0(0)$ & $0(0)$ & $21(100)$ & -5.3613 \\
\hline
\end{tabular}

${ }^{*}$ See note on Table 2 .

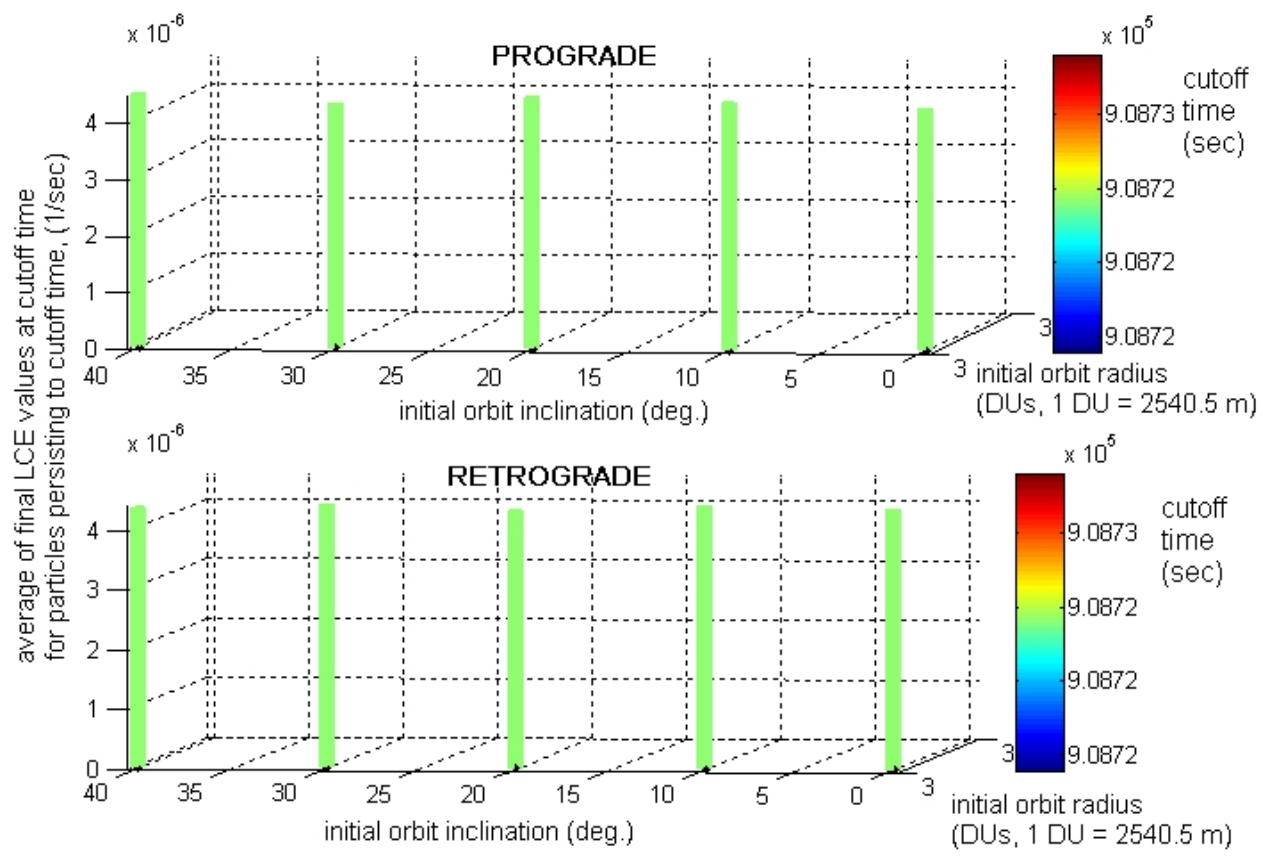

Figure 6. Graphical illustration of cutoff duration and average LCE value at that duration for barycentric orbits, both prograde and retrograde, at farthest average distance from the binary barycenter.

paths staying roughly in the plane of the binary's mutual orbit, but the results observed here indicate a need to also incorporate the $\pm \mathrm{Z}$ direction. A higher inclination orbit about Alpha is likewise beneficial for angular coverage and mapping of not only Alpha, but the polar regions of Beta as well.

We observe that in each Alpha-centric case there is also a middle range in the initial radius value sampled that produces the least instability. This is most apparent from Figure 2, where for the largest initial radius value the LCE vs. time curves do not drop below the minimum for those curves reached in the previous case with smaller radius. In fact, it appears that the best radius is at or just outside of the one to produce a 3:1 resonance between the particle and the binary system orbit periods. 
Beta-centric orbits in general appear to be more easily disrupted than orbits about Alpha, and almost always degrade comparatively rapidly, at least when started with the sampled radius values used herein and assuming matching circular speed for the initial velocity. This likely means that a significantly different initial velocity condition needs to be selected within the context of the three body problem. Note that in our example system, or in any member of the general class of binary NEAs formed by spin-up to fission, Beta is not rotating as rapidly as Alpha nor rotating at all with respect to the usual three body problem frame. This may contribute to the quicker trajectory degradation about Beta. Note how for prograde Beta-centric orbits at a fixed radius the performance improves dramatically with increasing inclination, then drops, and then improves again. However, retrograde Beta-centric paths at the same fixed radius seem to follow a trend in stability performance vs. inclination going in the opposite sense of the trend for prograde paths.

Finally, far-field orbits about the system barycenter unsurprisingly exhibit much greater stability than either the Alpha-centric or Beta-centric paths within the system. However in this case it is seen that there is little change with increasing inclination and also little difference between the retrograde and prograde orbit types. Perhaps differences in duration of the particle trajectories prior to impact or escape would become apparent between the orbit types and various inclinations if the duration of propagation for the test particles were not limited to the length of the underlying full two body problem propagation, which is only a little over two weeks. In this sense, the flight time for the test particles in these far-field orbits seems to be saturated at an artificial limit.

\section{Methodology for Local Instability Exploration}

We would like to improve the stability of the nominal trajectory in each case examined above, by using an initial condition which generates a nominal trajectory which is closer to periodic, i.e. closer to repeating the same location in the Poincaré section formed by the plane of syzygy. (This is the plane formed by the instantaneous line of syzygy and binary's instantaneous mutual orbit normal.) In particular, we refine the initial velocity while keeping the initial position fixed. We do this starting with our prior guess of velocity direction perpendicular to the initial radial position vector from the orbit center, with the given inclination, and velocity magnitude matching the circular orbit speed. (For which the orbit center is just considered as an idealized point mass for each body, or the total system mass at the system barycenter.) We choose to vary the velocity magnitude but not the direction, first changing the magnitude with a linear sweep covering $\pm 9 \%$ of the nominal value in $1 \%$ increments.

For the propagation of this sweep of trajectories, and some other trajectories mentioned below, we implement a switch in our restricted full three body problem propagation code to detect crossing of the syzygy plane and stop integration at such a crossing. Prior to the update portion of the RKF7(8) integration algorithm, we perform instead a virtual update and check what the state would become with that and also what the new binary state would become, with the passage of the current time step. Unit vectors of the instantaneous plane of syzygy frame matching these virtually updated particle and binary states are found, and the particle position and velocity vectors are decomposed into components in that frame. If the $+\mathrm{Y}$ component of position in this frame (the component in the direction of the mutual orbit normal crossed into the line from Alpha to Beta) changes sign relative to its previous value upon this virtual update, then the actual update is rejected and the usual RKF7(8) step size adjustment is overridden by a halving of the current step size. This is all repeated before the actual update portion of the algorithm is reached again, and so on. Figure 7 shows first, at top, the simulation time left until the exact time of reaching the syzygy plane (as defined later by the plane crossing time eventually converged to) before each update that was not adopted because an overshoot of the plane was detected. At the bottom in the same figure is shown the $+\mathrm{Y}$ component of position in this frame, both before (blue) and after (red) each update that was not adopted because an overshoot of the plane was detected. The count of such skipped updates forms the independent variable axis in these plots. Figure 7 demonstrates that we are accurately determining the instant and conditions at plane crossing, and propagating up to that instant through the minimum step size being reached, thus stopping the integration.

For a given particle trajectory, all components of the position and velocity expressed in the syzygy frame at the time of next plane crossing, when the propagation stops, can be differenced with the position and velocity expressed in the syzygy frame at the initial time. This gives the position and velocity deviations $\delta \mathbf{d}$ 

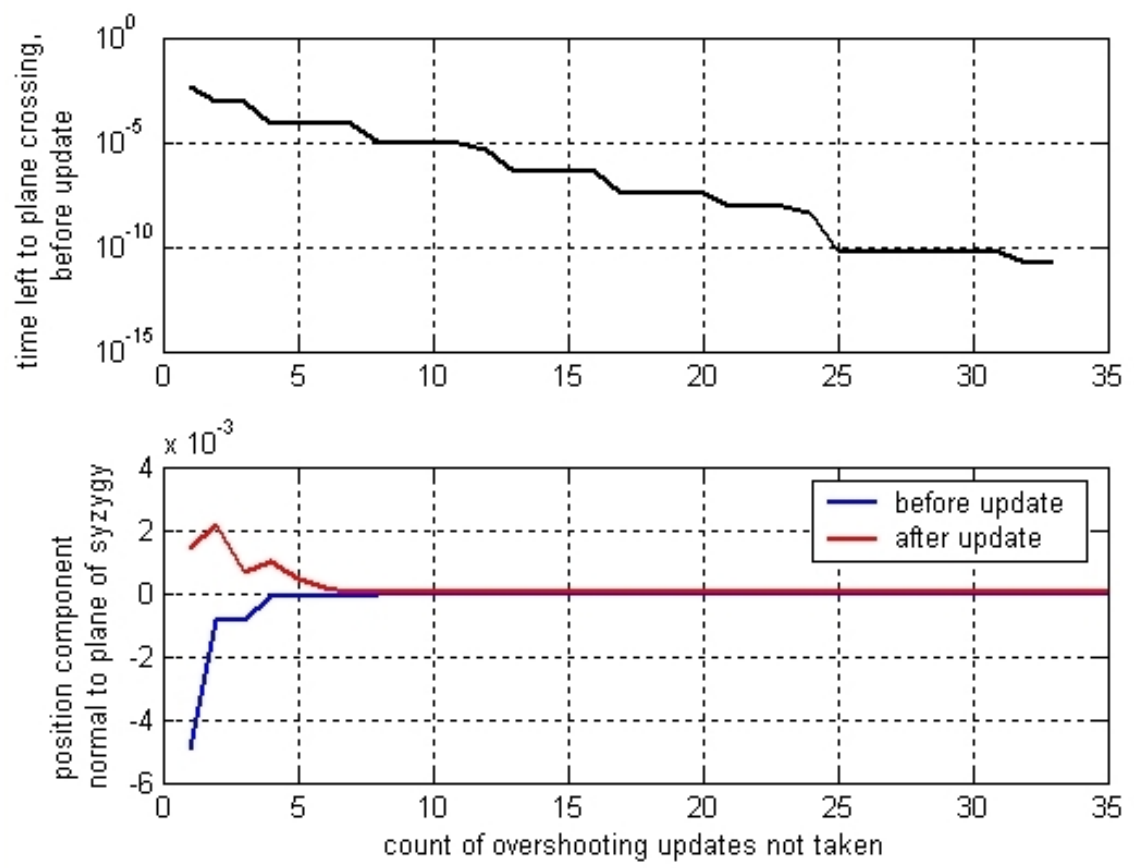

Figure 7. Performance in converging to exact time of syzygy plane crossing, for accurate formulation of cost functional.

and $\delta \mathbf{v}$ for use in the cost functional

$$
J=\frac{1}{2}(\delta \mathbf{d} \cdot \delta \mathbf{d})+\frac{1}{2}(\delta \mathbf{v} \cdot \delta \mathbf{v})
$$

From our sweep of initial velocity magnitudes, the velocity magnitude value resulting in the lowest $J$ is adopted to define a new particle initial state serving as the starting point for iterative refinement. For the iteration, we must first initialize our simple algorithm. This is done by propagating to the next syzygy plane crossing new trajectories having initial velocity magnitude $5 \%, 10 \%$, and $0 \%$ above the iteration starting point. Once these three trajectories and their end states and corresponding costs are in hand, we use the following to perform the actual iteration:

$$
\|\mathbf{v}\|_{i+1}=-\frac{b_{i}}{c_{i}} \quad, \quad\left[\begin{array}{c}
a_{i} \\
b_{i} \\
c_{i}
\end{array}\right]=\left[\begin{array}{ccc}
1.0 & \|\mathbf{v}\|_{i-2} & \frac{1}{2}\|\mathbf{v}\|_{i-2}^{2} \\
1.0 & \|\mathbf{v}\|_{i-1} & \frac{1}{2}\|\mathbf{v}\|_{i-1}^{2} \\
1.0 & \|\mathbf{v}\|_{i} & \frac{1}{2}\|\mathbf{v}\|_{i}^{2}
\end{array}\right]^{-1}\left[\begin{array}{c}
J_{i-2} \\
J_{i-1} \\
J_{i}
\end{array}\right]
$$

With each iteration, $i$ is incremented and the newest cost $J_{i}$ for the end state of the last propagation, in turn started using $\|\mathbf{v}\|_{i}$, is incorporated.

From all of the iterated values used for initial velocity magnitude, again the value resulting in the lowest $J$ is adopted to define the initial state for an improved nominal trajectory. This is propagated for the full duration with no syzygy plane crossing detection enabled, with the intent of being able to directly compare the duration reached and long-term LCE value reached between the improved nominal trajectory and the old nominal trajectory, within the same region-type-radius-inclination case.

All of the steps outlined in this section are applied only to a small subset of the full set of such cases. In particular, we do these steps for the Alpha-centric prograde orbits at the lowest radius, highest radius, and radius from our sampling which is nearest to that for the (possibly least unstable) 3:1 resonance between the particle and binary system orbital periods. For these three radii, all inclination values are explored. 


\section{Local Exploration Results}

Here we compare the best results obtained with our linear sweep and subsequent iteration in the scalar parameter $\|\mathbf{v}\|$ against the original results obtained, for the nominal trajectories in our subset of cases of particular interest. Figures 8-10 show this comparison in the LCE vs. time curves from the propagated trajectories, with the dotted lines being for the "improved" nominal trajectories and solid lines being for the original ones.

It is seen that while the winner of this comparison varies considerably between cases, with the newer nominal trajectory sometimes performing better and sometimes performing worse, in general there is no net improvement in stability metric performance resulting from the computational effort of our $\|\mathbf{v}\|$ parameter exploration. Over the short (less than about 6 hours) lifetimes for the trajectories starting closest to Alpha's surface, almost no change is observable. Average lifetimes are still about the same for the trajectories starting at the larger radii, with the same $40^{\circ}$ inclined trajectory persisting longest (and separated by only a couple days in favor of the original initial condition for the case nearest the hypothesized stable resonance). Remedies for this failure to achieve performance improvement may include varying a larger number of initial state components or parameters, to work with more degrees of freedom. Also, it may help to use a more sophisticated gradient-based optimization for the cost functional rather than the simple iterative scheme proposed above (which depends on the cost being approximately locally quadratic in the single varied parameter of initial velocity magnitude). Of course another explanation for the lack of improvement may be that the stability properties of this dynamical system simply do not change strongly with location in phase space, in which case the approach used will have little impact.

\section{Conclusions and Future Work}

In this work we have taken first steps towards global characterization of the degree of instability of trajectories within and about any member of the majority class of binary NEA systems. Our simulation of test particles in the restricted full three body problem, for an example system which typifies that class, has explored a limited parameter space governing the choice of trajectory initial conditions. In particular, we have explored trajectories about both components and about the system as a whole, in both directions with

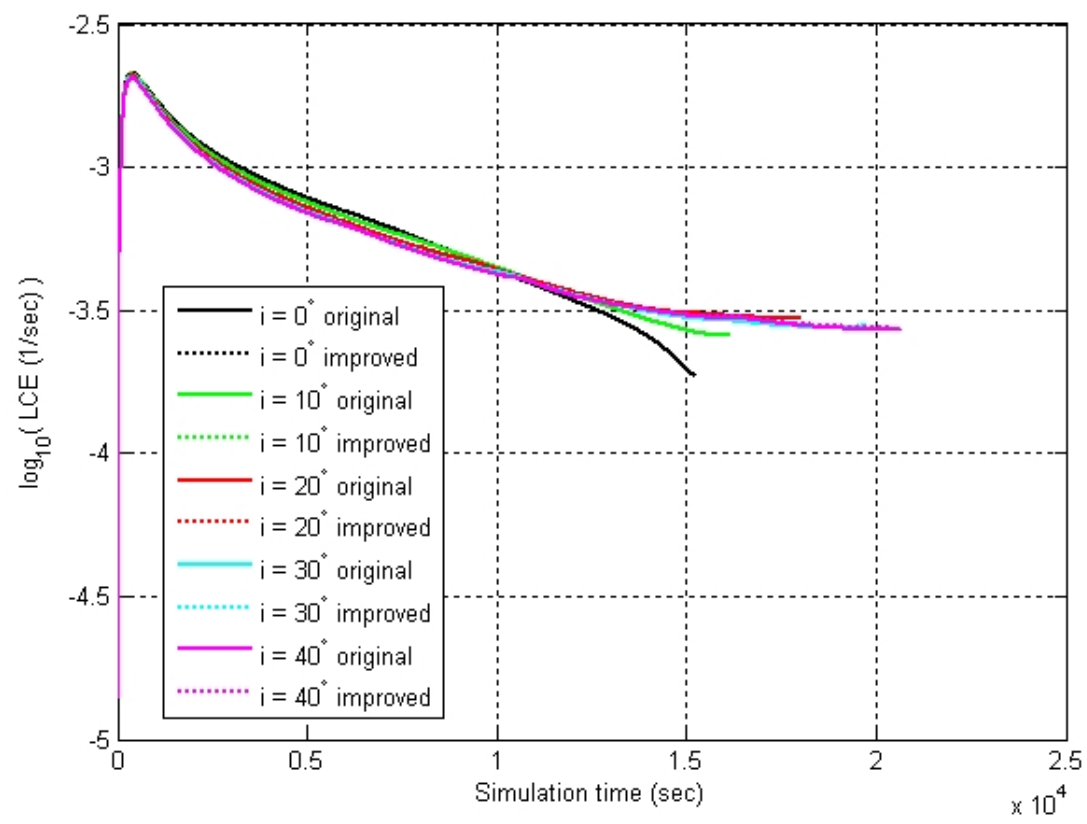

Figure 8. Comparison of the LCE plotted against simulation time between original nominal trajectories (solid lines) and the "improved" nominal trajectories produced with our sampling and iteration in the initial velocity magnitude parameter (dotted lines). All of this is for the Alpha-centric, prograde, $R_{o}=0.3397$ cases. 


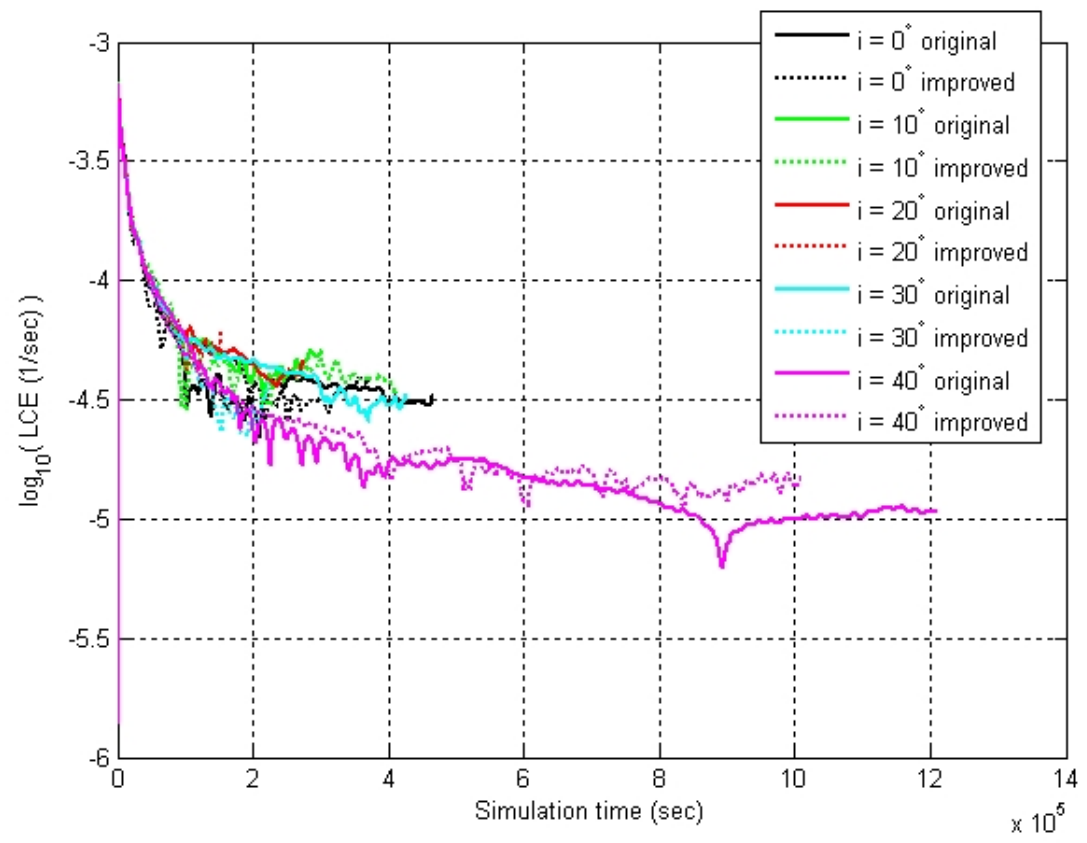

Figure 9. Comparison of the LCE plotted against simulation time between original nominal trajectories (solid lines) and the "improved" nominal trajectories produced with our sampling and iteration in the initial velocity magnitude parameter (dotted lines). All of this is for the Alpha-centric, prograde, $R_{o}=0.50389$ cases.

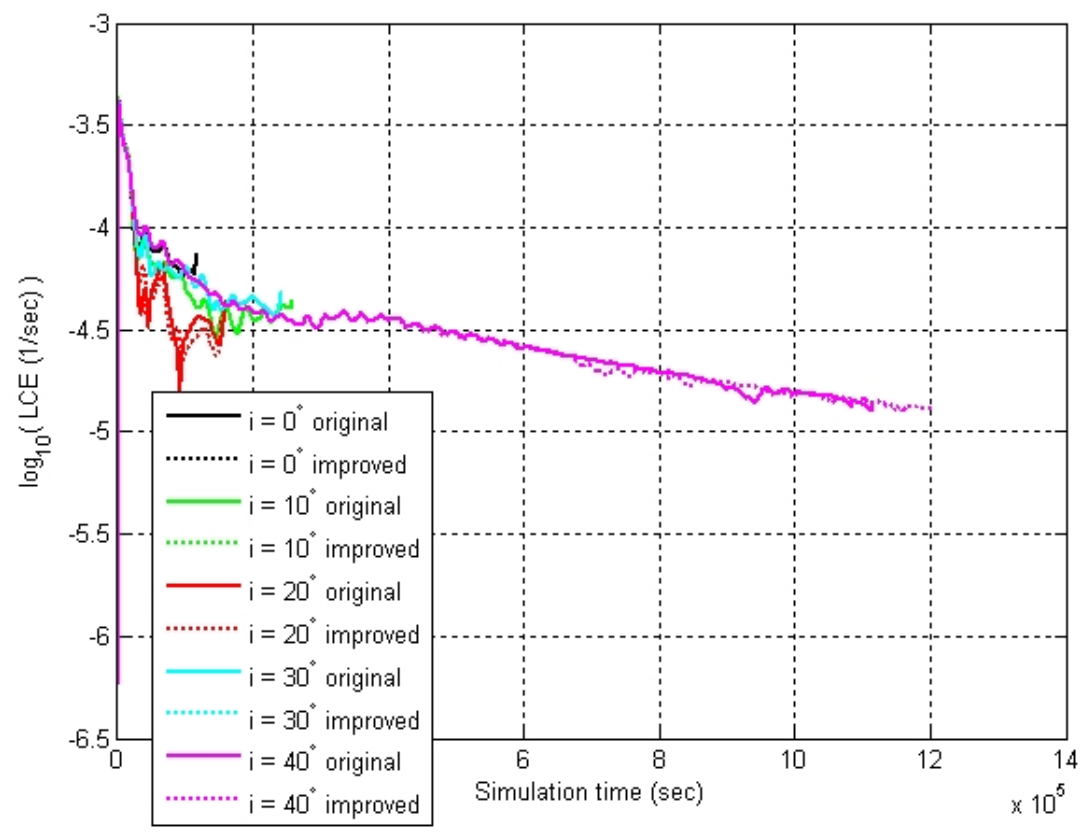

Figure 10. Comparison of the LCE plotted against simulation time between original nominal trajectories (solid lines) and the "improved" nominal trajectories produced with our sampling and iteration in the initial velocity magnitude parameter (dotted lines). All of this is for the Alpha-centric, prograde, $R_{o}=0.61335$ cases.

respect to the three body problem frame's mean rotation, with varying scaled proximity to either component plus varying initial departure from the instantaneous plane of the binary mutual orbit. For all test particles, we have tracked their impact vs. escape outcomes, but primarily we have focused on determining the finite- 
time LCE's of their trajectories as the metric for how unstable those trajectories are. Although we used a direct sampling and then iterative scheme to attempt to refine a single initial condition parameter (initial velocity magnitude) to improve trajectory duration and performance according to this metric, this produced minimal benefit. Further pursuing the orbit refinement path is advisable only with improvements over our methods.

Obviously there are many still unexplored directions in which this work can be expanded. In addition to the options mentioned earlier for changing our methodology for finding improved nominal trajectories, we can apply that methodology to all of the cases in our parameter space, rather than just a subset of them. Also, it may be fruitful to expand the length of the underlying full two body problem propagation results, to allow for longer particle trajectory durations so that saturation at an artificial limiting duration is encountered less frequently. This may allow cases with virtually indistinguishable outcomes in the current results to become differentiated. Additionally, one may propagate a far larger set of randomly deviated perturbed particles about each nominal trajectory initial condition. This would give sufficient density to a mapping of the individual (not averaged) particle LCE values onto the dimensions of random initial state deviation in the phase space. A sufficient density of points over a narrow span in such a mapping may allow a fit for local gradients of stability.

Several insights into the best trajectories within a binary system to use as part of a future scientific mission to such a pair can still be gained from this work in its present state. Orbiting at a distance from the barycenter a few times larger than the separation between the binary component mass centers is the most natural starting point. The low LCE values for such orbits indicate that this choice is good for the goal of flight safety and long observation time at a larger distance, up to at least a few weeks. However, this supposes that the binary as a whole is large enough or dense enough that it's net gravitational force on the spacecraft at this distance is at least an order of magnitude larger than the force on the spacecraft from solar radiation pressure (SRP), which was not incorporated into any propagation within this paper. The observed insensitivity of stability performance with respect to inclination from the binary's mutual orbit plane means a higher inclination, up to $40^{\circ}$, can and should be chosen. This serves the goal of bettering the angular coverage in latitude on both bodies, for imaging, spectroscopy, or point-ranging measurement mapping of the surfaces. Further, bearing in mind the actual presence of SRP, this inclination should be selected to place the spacecraft's orbit as close to within the terminator plane perpendicular to the direction of the sun as possible. The direction of the spacecraft orbit should also be retrograde to accommodate transfer into the best choice for close proximity flight within the system, to accomplish detailed gravity mapping and the highest resolution surface observations. This would be a retrograde orbit that is significantly inclined (helpful for SRP and for Alpha mapping, as well as observing of high latitudes on Beta) and with an orbital radius from Alpha's center of mass about $1 / 2$ the separation between the component mass centers. To obtain the most information about the gravity field and density distribution of the components it seems desirable to persist in these orbits (though they are the safest rather than the most strongly influenced paths) for as long as possible without any trajectory correction thruster actuation. These general guidelines supported by the results of this study should be useful for the future design of binary asteroid missions.

\section{Acknowledgments}

E.G.F. would like to acknowledge support under a National Science Foundation Graduate Research Fellowship for conducting this work. D.J.S. acknowledges support from a NASA grant from the Planetary Geology and Geophysics program. Supercomputers used for limited portions of this work were provided by funding from the JPL Office of the Chief Information Officer.

\section{References}

\footnotetext{
${ }^{1}$ Bottke Jr., W. F. and Melosh, H. J., "The Formation of Binary Asteroids and Doublet Craters," Icarus, Vol. 124, 1996, pp. 372391.

${ }^{2}$ Bottke Jr., W. F. and Melosh, H. J., "The Formation of Asteroid Satellites and Doublet Craters by Planetary Tidal Forces," Nature, Vol. 381, 1996, pp. 5153.

${ }^{3}$ Margot, J. L., Nolan, M. C., Benner, L. A. M., Ostro, S. J., Jurgens, R. F., Giorgini, J. D., Slade, M. A., and Campbell, D. B., "Binary Asteroids in the Near-Earth Object Population," Science, Vol. 296, May 2002, pp. 1445-1448.

${ }^{4}$ Merline, W. J., Weidenschilling, S. J., Durda, D. D., Margot, J. L., Pravec, P., and Storrs, A. D., "Asteroids do have satellites," Asteroids III, edited by W. F. Bottke Jr., A. Cellino, P. Paolicchi, and R. Binzel, Space Science Series, Univ. of Arizona Press, Tucson, 2002, pp. 289-312.
} 
${ }^{5}$ Fahnestock, E. G. and Scheeres, D. J., "Simulation and analysis of the dynamics of binary Near-Earth Asteroid (66391) 1999 KW4," Icarus, Vol. 194, No. 2, April 2008, pp. 410-435.

${ }^{6}$ Ostro, S. J., Margot, J.-L., Benner, L. A. M., Giorgini, J. D., Scheeres, D. J., Fahnestock, E. G., Broschart, S. B., Bellerose, J., Nolan, M. C., Magri, C., Pravec, P., Scheirich, P., Rose, R., Jurgens, R. F., De Jong, E. M., and Suzuki, S., "Radar Imaging of Binary Near-Earth Asteroid (66391) 1999 KW4," Science, Vol. 314, Nov. 2006, pp. 1276-1280.

${ }^{7}$ Scheeres, D. J., Fahnestock, E. G., Ostro, S. J., Margot, J.-L., Benner, L. A. M., Broschart, S. B., Bellerose, J., Giorgini, J. D., Nolan, M. C., Magri, C., Pravec, P., Scheirich, P., Rose, R., Jurgens, R. F., De Jong, E. M., and Suzuki, S., "Dynamical Configuration of Binary Near-Earth Asteroid (66391) 1999 KW4," Science, Vol. 314, Nov. 2006, pp. 1280-1283.

${ }^{8}$ Borderies, N., "Mutual Gravitational Potential of N Solid Bodies," Celestial Mechanics, Vol. 18, No. 3, 1978, pp. $295-307$.

${ }^{9}$ Braun, C. V., The Gravitational Potential of Two Arbitrary, Rotating Bodies with Applications to the Earth-Moon System, Ph.D. thesis, University of Texas at Austin, 1991.

${ }^{10}$ Werner, R. A. and Scheeres, D. J., "Mutual Potential of Homogenous Polyhedra," Celestial Mechanics and Dynamical Astronomy, Vol. 91, No. 3, March 2005, pp. 337-349.

${ }^{11}$ Fahnestock, E. G. and Scheeres, D. J., "Simulation of the Full Two Rigid Body Problem Using Polyhedral Mutual Potential and Potential Derivatives Approach," Celestial Mechanics and Dynamical Astronomy, Vol. 96, No. 3-4, Nov. 2006, pp. 317-339.

${ }^{12}$ Werner, R. A. and Scheeres, D. J., "Exterior Gravitation of a Polyhedron Derived and Compared with Harmonic and Mascon Gravitation Representations of Asteroid 4769 Castalia," Celestial Mechanics and Dynamical Astronomy, Vol. 65, No. 3, 1997, pp. 313-344. 